\title{
ON ROOTS AND SUBSEMIGROUPS OF NILPOTENT GROUPS
}

\author{
JOSEPH E. KUCZKOWSKI
}

\begin{abstract}
E_{\omega}, U_{\omega}$ and $D_{\omega}$ semigroups are defined by extrapolating the definitions of their group counterparts; and a class $n$ semigroup is defined to be a subsemigroup of a class $n$ group. The purpose of this paper is to show that a class $n E_{\omega}$ semigroup generates an $E_{\omega}$ group and that a class $n$ semigroup is $U_{\omega}$ if and only if it generates a $U_{\omega}$ group.
\end{abstract}

$E_{\omega}, U_{\omega}$, and $D_{\omega}$ semigroups are defined in a manner similar to the group definitions presented by G. Baumslag [1]. Thus, let $\omega$ be a nonempty fixed set of primes. A semigroup $S$ is an $E_{\omega}$ semigroup if for any $s \in S$ and $p \in \omega$ there exists $t \in S$ so that $s=t^{p}$. $S$ is a $U_{\omega}$ semigroup if $p$ th roots are unique when they exist. If $S$ is both $E_{\omega}$ and $U_{\omega}$, then $S$ is called a $D_{\omega}$ semigroup.

The properties of existence and uniqueness of roots are investigated with respect to subsemigroups of class $n$ nilpotent groups (referred to as class $n$ semigroups) and the subgroups which these semigroups generate.

The purpose of this paper is to prove the following two theorems:

TheOREM A. A class $n$ semigroup is a $U_{\omega}$ semigroup if and only if it generates a $U_{\omega}$ group.

TheOREM B. $A$ class $n E_{\omega}$ semigroup generates an $E_{\omega}$ group.

Remark 1. The converse of Theorem B is not available. Consider, for example, the semigroup $Q^{\prime}$ of rational numbers $q, q \geqq 1$, under addition. $Q^{\prime}$ is not divisible, but it generates the divisible group of additive rationals.

REMARK 2. Referring to the paper of B. H. Neumann and T. Taylor [3] on subsemigroups of nilpotent groups, Theorems A and B may be recast as follows:

ThEOREM $\mathrm{A}^{\prime}$. A cancellative semigroup satisfying the $L_{n}$ law is $U_{\omega}$ if and only if it generates a class $n U_{\omega}$ group.

ThEOREM B'. An $E_{\omega}$ cancellative semigroup satisfying the $L_{n}$ law generates a class $n E_{\omega}$ group.

Received by the editors April 15, 1970.

AMS 1969 subject classifications. Primary 2040, 2092, 2093.

Key words and phrases. $E_{\omega}$ semigroup, $U_{\omega}$ semigroup, $D_{\omega}$ semigroup, $\omega$-free group, w-subgroup. 


\section{Proof of Theorem A.}

A1. If $S$ is a class $n U_{\omega}$ semigroup in a group $G$, then the center of the group generated by $S, Z(\operatorname{gp}\{S\})$, is $\omega$-free i.e. having no elements of order $p$ for any $p \in \omega$.

According to Neumann-Taylor [3], gp $\{S\}=S S^{-1}=S^{-1} S$. Thus, suppose $s_{1} s_{2}^{-1} \in Z(\operatorname{gp}\{S\})$ for some $s_{1}, s_{2} \in S$ and that $\left(s_{1} s_{2}^{-1}\right)^{p}=1$, where $p$ is some element of $\omega$ and 1 is the identity of $G$. Now, $s_{1}$ and $s_{2}$ commute so that $s_{1}^{p}=s_{2}^{p}$. Since $S$ is $U_{\omega}, s_{1}=s_{2}$ and $s_{1} s_{2}^{-1}=1$.

A2. If $S$ is a class $n U_{\omega}$ semigroup in a group $G$, then $\operatorname{gp}\{S\}$ is $\omega$-free.

Suppose the upper central series for $\operatorname{gp}\{S\}$ is $\{1\}=Z_{0} \leqq Z_{1} \leqq \ldots$ $\leqq Z_{n}=\operatorname{gp}\{S\} . Z_{1}$ is the center of $\operatorname{gp}\{S\}$ and, according to part A1, is $\omega$-free. Since $Z_{1}$ is abelian, it is clearly a $U_{\omega}$ subgroup so that the identity subgroup $Z_{0}$ is an $\omega$-subgroup of $Z_{1}$. Here we may recall that a subgroup $H$ of a group $K$ is called an $\omega$-subgroup of $K$ if the relation $k^{p} \in H$ implies $k \in H$ for any pair $k$ and $p$, with $k \in K$ and $p \in \omega$.

Let us proceed by induction and assume for each pair $\left\{Z_{i-1}, Z_{i}\right\}$, $i=1, \cdots, k-1$, that $Z_{i}$ is $\omega$-free and $Z_{i-1}$ is an $\omega$-subgroup of $Z_{i}$. It may then be shown that for $i=k$, the pair $\left\{Z_{k-1}, Z_{k}\right\}$ fulfills the conditions just described. Following the usual argument we conclude that $Z_{n}=\operatorname{gp}\{S\}$ is $\omega$-free.

Now, suppose $s \in Z_{k}$ and $s^{p}=1$ for some $p \in \omega$. Then $1=\left[s^{p}, t\right]$ for each $t \in \operatorname{gp}\{S\} .\left[s^{p}, t\right]=\left[s^{p-1}, t\right]^{s}[s, t]=\left[s^{p-1}, t\right]\left[\left[s^{p-1}, t\right], s\right][s, t]$. However, $\left[s^{p-1}, t\right] \in Z_{k-1}$ so that $\left[\left[s^{p-1}, t\right], s\right] \in Z_{k-2}$. Thus, $1=\left[s^{p}, t\right]$ $=\left[s^{p-1}, t\right][s, t] s_{1}$ where $s_{1} \in Z_{k-2}$. Proceeding in this way we finally have $1=\left[s^{p}, t\right]=[s, t]^{p} s_{2}$ for some $s_{2} \in Z_{k-2}$. But, under the induction assumption, $Z_{k-2}$ is an $\omega$-subgroup of $Z_{k-1}$ so that $[s, t] \in Z_{k-2}$ and $s$ must now be an element of $Z_{k-1}$, which is assumed to be $\omega$-free. Hence, $s^{p}=1$ implies $s=1$ and $Z_{k}$ is $\omega$-free.

We follow a similar argument to show that $Z_{k-1}$ is an $\omega$-subgroup of $Z_{k}$.

A3. A class $n$ semigroup is $U_{\omega}$ if and only if $\operatorname{gp}\{S\}$ is $\omega$-free.

Part A2 tells us that $\mathrm{gp}\{S\}$ is $\omega$-free if $S$ is a $U_{\omega}$ semigroup. On the other hand, suppose $S$ is not $U_{\omega}$. Then there exist $x, y \in S, x \neq y$, and $p \in \omega$ with $x^{p}=y^{p}$. But, following a statement of P. Hall [2], $x y^{-1}$ has order dividing a power of $p$. Consequently, $\operatorname{gp}\{S\}$ is not $\omega$-free.

The following corollary of A3 is an important statement on $U_{\omega}$ groups and is attributed to Mal'cev and Cernikov in Baumslag [1].

A4. A nilpotent group $G$ is a $U_{\omega}$ group if and only if it is $\omega$-free.

A5. Theorem A. A class $n$ semigroup is a $U_{\omega}$ semigroup if and only if it generates a $U_{\omega}$ group. 
It is clear that if $S$ is a subsemigroup of a $U_{\omega}$ group then $S$ is also $U_{\omega}$. The converse is obtained as a consequence of parts A3 and A4.

ProOF OF THEOREM B.

B1. If $S$ is an $E_{\omega}$ subsemigroup of a commutative group, then $H=\operatorname{gp}\{S\}$ is $E_{\omega}$.

Since gp $\{S\}=S S^{-1}$, consider $x y^{-1} \in \operatorname{gp}\{S\}$ for some $x, y \in S$ and fix $p \in \omega$. Then, there exist $x_{1}$ and $y_{1}$ belonging to $S$ so that $x_{1}^{p}=x$ and $y_{1}^{p}=y$. Thus, $x y^{-1}=\left(x_{1} y_{1}^{-1}\right)^{p}$ and the conclusion follows.

B2. Theorem B. A class $n E_{\omega}$ semigroup generates an $E_{\omega}$ group.

Having verified the theorem in the abelian case in part B1, we proceed by induction and assume for class $n>1$ that the conclusion is valid for $E_{\omega}$ semigroups of class less than $n$.

Let $H=\operatorname{gp}\{S\}$ have lower central series $H=H^{1} \geqq H^{2} \geqq \cdots$ $\geqq H^{n+1}=\{1\} . H / H^{n}$ and, consequently, $S / H^{n}$ have class less than $n$. $S / H^{n}$ is clearly $E_{\omega}$ so that by the induction assumption $H / H^{n}$ is an $E_{\omega}$ group. Thus, for $h \in H$ and $p \in \omega$, there exists $h_{1} \in H$ so that $h H^{n}=\left(h_{1} H^{n}\right)^{p}$. It follows that $h=h_{1}^{p} z$ for some $z \in H^{n}$. We know that $H^{n}$ is generated by all transforms in $H$ of commutators of the form $\left[s_{1}, \cdots, s_{n}\right]$, where $s_{i} \in S, i=1, \cdots, n,[2]$.

Consider the commutator mentioned above. There is a $t \in S$ so that $s_{n}=t^{p}$. Thus, $\left[s_{1}, \cdots, s_{n-1}, s_{n}\right]=\left[s_{1}, \cdots, s_{n-1}, t^{p}\right]=\left[s_{1}, \cdots, s_{n-1}, t\right]^{p}$. We see that every transform of the commutators under consideration has $p$ th roots in $H^{n}$. But $H^{n}$ is also central and thus every element of $H^{n}$ has a $p$ th root in $H^{n}$.

Let $z_{1} \in H^{n}$ be a $p$ th root of $z$. Then $h=h_{1}^{p} z=\left(h_{1} z_{1}\right)^{p}$; and $H$ is an $E_{\omega}$ group.

Theorems A and B yield the following:

Corollary C. If $S$ is a class $n D_{\omega}$ semigroup then $S$ generates a $D_{\omega}$ group.

Corollary $C^{\prime}$. If $S$ is a cancellative $D_{\omega}$ semigroup satisfying the $L_{n}$ law, then $S$ generates a class $n D_{\omega}$ group.

\section{REFERENCES}

1. G. Baumslag, Some aspects of groups with unique roots, Acta Math. 104 (1960), 217-303. MR 23 \#A191.

2. P. Hall, Nilpotent groups, Canadian Mathematical Congress Summer Seminar, University of Alberta, 1957.

3. B. H. Neumann and T. Taylor, Subsemigroups of nilpotent groups, Proc. Roy. Soc. Ser. A 274 (1963), 1-4. MR 28 \#3100.

PURDUE UNIVERSITy, INDIANAPOLIS, INDIANA 46205 